\title{
Design of Peptide Nanoparticles Using Simple Protein Oligomerization Domains
}

\author{
Senthilkumar Raman ${ }^{1}$, Gia Machaidze ${ }^{1}$, Ariel Lustig ${ }^{1}$, Vesna Olivieri, Ueli Aebi ${ }^{1}$ and \\ Peter Burkhard*,1,2 \\ ${ }^{1}$ M.E. Müller Institute for Structural Biology, Biozentrum, University of Basel, 4056 Basel, Switzerland \\ ${ }^{2}$ The Institute of Materials Science, University of Connecticut, Storrs, CT 06269-3136, USA
}

\begin{abstract}
Viruses are naturally formed bionanoparticles ranging in size from about 20 to $150 \mathrm{~nm}$. Remarkably, small viruses are composed of one single protein chain folding into a capsid structure with icosahedral symmetry. The icosahedron is built from 60 asymmetric units and is the largest closed shell in which every subunit is in an identical environment. It is characterized by 2 -fold, 3 -fold and 5-fold rotational symmetry axes. By superposition of different protein oligomerization domains onto the symmetry axes of an icosahedron, a nanoparticle with icosahedral symmetry can be designed. We have recently described such a design of peptide nanoparticles using coiled-coil protein oligomerization domains. Here we show that oligomerization motifs other than coiled-coils can be used to form nanoparticles by incorporating the globular foldon domain from fibritin with a trimeric $\beta$-sheet conformation into the design. We expressed and purified 8 different peptides and performed refolding studies and biophysical characterization with analytical ultra centrifugation (AUC) and electron microscopy (EM). In the first design version we joined the foldon domain to the pentameric coiled-coil domain of COMP and varied the lengths of the linker sequences between the two domains. In this design we observed only smaller nanoparticles. When in the second design the foldon domain was extended with an additional trimeric coiled-coil domain as a combined trimerization domain that is linked to the COMP pentamer, we observed nanoparticles of sizes and molecular weights as would be expected for icosahedral symmetry. Viruses and virus-like particles (VLPs) are known for their ability to induce a strong humoral and hence antibody mediated immune response due to their repetitive antigen display. Peptide based nanoparticles have similar properties to VLPs, which are in clinical trials as a carrier in vaccination. Therefore, these peptide nanoparticles represent an alternative platform for subunit vaccine using the concept of repetitive antigen display.
\end{abstract}

Keywords: Nanoparticle, protein design, coiled-coil, foldon, vaccine design, repetitive antigen display.

\section{INTRODUCTION}

Nanotechnology is exploring the "plenty of room at the bottom" that Richard Feynman referred to nearly half a century ago [1], when reasoning about the possibilities of manipulating matter at the atomic and molecular scales. Much research has since been done leading to a wide variety of biomedical applications for nanostructures and nanoparticles. For example, dendrimers [2,3] and magnetic nanoparticles $[4,5]$ can be used to target cancer cells and in tissue imaging; gold nanoshells [6] can be used in cancer therapy; liposomes $[7,8]$ have applications in gene therapy and drug delivery. However, with the growing number of applications of inorganic, metallic, semiconductor and polymer based nanoparticles in biology, there is also increasing concern about the toxicological properties of these nanoparticles. For example, carbon nanoparticles have been shown to induce lipid per-oxidation in the brain cells of fish and pulmonary inflammation in rats $[9,10]$. Therefore, as an alternative researchers started using peptides and proteins as nanoparticle building blocks. This has opened up a new field of research called nano-biotechnology, which focuses on the research

*Address correspondence to this author at the Institute of Materials Science, University of Connecticut, Storrs, CT 06269-3136, USA; Tel: +860 486 3830; Fax: +860 486 4745; E-mail: peter.burkhard@uconn.edu and development of novel nanoscale materials made from protein building blocks for medical applications such as gene delivery, bio-imaging, drug delivery and vaccine development.

Self-assembly is one of the processes used in nanotechnology to generate supramolecular nanostructures [11], and in particular, self-assembly processes are exploited to create novel nanoscale biomaterials. Nature itself provides a rich source of self-assembled nanoscale structures such as viruses, ribosomes and many other highly complex, supramolecular machineries. By combining molecular biology and recombinant protein expression, several self-assembled nanoscale structures derived from nature's repertoire have been prepared for nanobiotechnological applications, including virus-like particles (VLPs) [12,13], ferritin protein cages [14-16], heat shock protein cages [17-19] and other selfassembled protein cages, such as enzyme complexes [20], chaperones [21], and carboxysomes [22]. According to Lee and Wang [23], the following characteristics make bionanoparticles more attractive compared to synthetic nanoparticles: they have well organized architectures with a broad selection of sizes at the nanometer scale; they are monodisperse with uniform sizes and shapes; their three-dimensional structures can be resolved at atomic or near-atomic resolution; and they can be economically produced at a large scale 
in gram or even kilogram quantities. Furthermore, through the availability of their gene sequence the composition and surface properties can be precisely controlled since they can be modified by both genetic and chemical techniques. Hence, their scaffolds can be altered and modified with near atomic precision.

More than 25 years ago Eric Drexler [24] suggested that protein design could be used as a "bottom-up" approach to fabricate devices by using peptides and proteins as monomeric building blocks to self-assemble into more complex, supramolecular structures. Still it is nearly impossible to design a protein scaffold completely from scratch: however, when using known protein folds such as coiled-coils as building blocks, things look quite different since a great deal is known about their folding and stability and the rules that govern their self-assembly into oligomeric structures.

Molecular symmetry can be used as a powerful tool in protein design for building large regular objects since proteins usually assume a unique and well-defined threedimensional architecture. Using this concept, Yeates and coworkers have exploited a general strategy for designing protein scaffolds that self-assemble into large symmetrical nanostructures, including protein cages, filaments, layers and porous materials [25].

We have recently developed a novel type of nanoparticles with icosahedral geometry (Fig. 1A) that self-assemble from 60 identical peptide chains [26]. The peptide chains are composed of a trimeric and pentameric oligomerization domain that are linked by a short peptide sequence. We call these particles self-assembling polypeptide nanoparticles (SAPN). In the design of Raman et al. 2006, the basic building blocks of the SAPN are coiled-coil motifs, which are the most frequently encountered oligomerization motifs in proteins [27-29]. Here, we wanted to investigate whether oligomerization motifs other than coiled coils can be used in an analogous design to form SAPN with icosahedral geometry. To accomplish this, we combined a non coiled-coil oligomerization motif (Foldon) together with either one or two coiled-coils (Laminin and COMP) as building blocks, and analyzed the self-assembly properties of these new SAPN. Depending on the building blocks contained in the constructs (Laminin, Foldon and COMP) we call the uncleaved versions of the constructs with all three building blocks LFC1 to LFC4, and the thrombin cleaved constructs with only two oligomerization domains FC1 to FC4 (see Fig. 2).

\section{MATERIALS AND METHODS}

\section{Cloning and Purification of LFC1 to LFC4 Constructs}

The oligonucleotides used to construct the LFC1 to LFC4 proteins were synthesized by Microsynth AG (Balgach, Switzerland), 5' phosphorylated and were quality assured by PAGE purification. All the oligonucleotides (both forward and reverse strand) of LFC1 to LFC4 constructs were dissolved in Milli-Q water to a concentration of $100 \mu \mathrm{M}$. The forward and reverse strand of each oligonucleotide were combined in $1 \mu \mathrm{M}$ concentration and $5 \mu \mathrm{l}$ of 20X SSC buffer (30 mM sodium citrate dihydrate $\mathrm{pH} 7.0$ and $300 \mathrm{mM} \mathrm{NaCl}$ for $100 \mathrm{ml}$ ) and water were added to give $50 \mu \mathrm{l}$. Annealing reaction was done in a thermocycler by first incubating at $90^{\circ} \mathrm{C}$ for $1 \mathrm{~min}$ followed by $70^{\circ} \mathrm{C}$ for $1 \mathrm{~min}$ and then cooling at $1 \% / 15 \mathrm{sec}$ to $20^{\circ} \mathrm{C}$ (Tpersonal, Biometra, UK) to give the annealed product. The annealed products were desalted using Micro Bio-Spin P-30 columns as per the manufacturers protocol (Bio-Rad, USA) and then checked in 4\% NuSieve GTG-Agarose (Cambrex Bio Science, USA) to confirm the annealing reaction. The annealed oligonucleotides of LFC1 to LFC4 were ligated into the multiple cloning site of the prokaryotic expression vector pPEP-T [30, 31]. Subsequently, the expression plasmids were transformed into $\mathrm{E}$. coli strain BL21 (DE3) pLysS cells (Novagen, USA).

A freshly transformed bacteria was inoculated in LB broth with ampicillin $(200 \mu \mathrm{g} / \mathrm{ml})$ and chloramphenicol (30 $\mu \mathrm{g} / \mathrm{ml}$ ) and grown overnight at $28^{\circ} \mathrm{C}$ with shaking at 180 rpm. $1 \%$ of the overnight grown culture was inoculated into fresh LB broth with ampicillin $(200 \mu \mathrm{g} / \mathrm{ml})$ and chloramphenicol $(30 \mu \mathrm{g} / \mathrm{ml})$ and incubated at $37^{\circ} \mathrm{C}$ with shaking at $180 \mathrm{rpm}$. Protein expression was induced when the cell density at $\mathrm{OD}_{600 \mathrm{~nm}}$ reached 0.5 by adding IPTG to a final concentration of $1 \mathrm{mM}$. After induction at $37^{\circ} \mathrm{C}$ for $3 \mathrm{hr}$, the cell pellet was collected by centrifugation at $6000 \mathrm{~g}$ for $20 \mathrm{~min}$ at $4{ }^{\circ} \mathrm{C}$ (Sorvall, GSA rotor). The bacterial cell pellets were thawed on ice and to each gram of pellet (wet weight), $3 \mathrm{ml}$ of lysis buffer pH 8.0 (8 M Urea, $10 \mathrm{mM}$ Tris-HCl, $100 \mathrm{mM}$ $\mathrm{NaH}_{2} \mathrm{PO}_{4}$ ) was added along with $10 \mathrm{mM} \beta$-mercaptoethanol. Pellets were thoroughly resuspended in lysis buffer and sonicated on ice for $5 \mathrm{~min}$ (4 sec of sonication and $6 \mathrm{sec}$ of gap) followed by centrifugation at $16000 \mathrm{rpm}$ at $4^{\circ} \mathrm{C}$ for $45 \mathrm{~min}$ to recover the supernatant from the cell debris. The resultant supernatant was mixed with $\mathrm{Ni}^{2+}$-NTA slurry (Qiagen, Switzerland) and gently shaked for $1 \mathrm{hr}$ at room temperature (batch method). After the incubation, the purification was done according to the protocol described by Qiagen and 10 $\mathrm{mM} \beta$-mercaptoethanol was included in all the buffers used during the purification step.

\section{Thrombin Cleavage and Purification of Cleaved Proteins FC1 to FC4}

The eluted proteins (LFC1 to LFC4) were refolded in a step wise dialysis under reducing conditions ( 2 mM DTT) by going from the $8 \mathrm{M}$ urea buffer containing $20 \mathrm{mM}$ Tris- $\mathrm{HCl}$ $\mathrm{pH} 8.4,150 \mathrm{mM} \mathrm{NaCl}$ to $6 \mathrm{M}$ urea followed by $4 \mathrm{M}$ and $2 \mathrm{M}$ and finally to $0 \mathrm{M}$ urea. After going to $0 \mathrm{M}$ urea, the protein samples were dialyzed against the buffer without DTT (20 $\mathrm{mM}$ Tris- $\mathrm{HCl} \mathrm{pH} 8.4,150 \mathrm{mM} \mathrm{NaCl}$ ). The refolded samples were dialyzed against the thrombin cleavage buffer $(20 \mathrm{mM}$ Tris- $\mathrm{HCl}(\mathrm{pH} 8.4), 150 \mathrm{mM} \mathrm{NaCl}$ and $\left.2.5 \mathrm{mM} \mathrm{CaCl}_{2}\right)$. For cleavage reactions, $1 \mathrm{U}$ of human plasma thrombin (Sigma, USA) was used per $\mathrm{mg}$ of protein and the reactions were carried out at room temperature for 16 to 18 hours or sometimes for 24 hours with gentle shaking. After cleavage reaction, the proteins were mixed with $\mathrm{Ni}^{2+}$-NTA slurry (Qiagen, Switzerland) and incubated at room temperature with gentle shaking for $1 \mathrm{hr}$ (batch method). Subsequently, the Ni-NTA column chromatography was done again to separate the cleaved proteins FC1 to FC4 (elution at $\mathrm{pH}$ 8.0) from the uncleaved proteins LFC1 to LFC4 and the His-tag along with laminin domain (mostly elution at $\mathrm{pH} 5.9$ ).

\section{SDS-PAGE and Western Blot}

Standard SDS-PAGE was performed [32] using Bio-Rad mini gel apparatus (Bio-Rad, USA). $15 \%$ and 17\% SDSPAGE were run to analyze the purified samples of LFC1 to 
LFC4 and FC1 to FC4 proteins, respectively. Proteins were visualized by Coomassie Brilliant blue staining. Estimates of molecular masses were obtained by comparison with lowmolecular-mass standards (GE Healthcare Life Sciences, range $94 \mathrm{kDa}$ to $14.4 \mathrm{kDa}$ ). Electrophoretic transfer of proteins from polyacrylamide gels to nitrocellulose membrane was done as described in the literature [33, 34]. Monoclonal antibody raised in mouse against the polyhistidine tag (Sigma, USA) was used to detect proteins containing Histag.

\section{Refolding Conditions}

The protein refolding procedure was carried out using two different refolding regimes. During refolding under both the regimes the protein concentration was kept at $0.1 \mathrm{mg} / \mathrm{ml}$.

\section{Regime 1 (Refolding Under Oxidizing Conditions)}

The purified protein in denaturing conditions was refolded by step wise dialysis procedure. Initially, the denatured protein was dialyzed against the buffer $20 \mathrm{mM}$ Tris$\mathrm{HCl} \mathrm{pH} 7.5,150 \mathrm{mM} \mathrm{NaCl}$ containing $8 \mathrm{M}$ urea followed by $6 \mathrm{M}$ urea, $4 \mathrm{M}$ urea, $2 \mathrm{M}$ urea and no urea in the same buffer as above. Finally, the protein was concentrated to the required concentration for further characterization in the buffer $20 \mathrm{mM}$ Tris- $\mathrm{HCl} \mathrm{pH}$ 7.5, $150 \mathrm{mM} \mathrm{NaCl}$.

\section{Regime 2 (Refolding Under Reducing Conditions)}

In this condition also, the step wise dialysis procedure was followed. Initially, the protein was dialyzed against the buffer $20 \mathrm{mM}$ Tris- $\mathrm{HCl} \mathrm{pH} 7.5,150 \mathrm{mM} \mathrm{NaCl}$ and $2 \mathrm{mM}$ DTT containing $8 \mathrm{M}$ urea followed by $6 \mathrm{M}$ urea, $4 \mathrm{M}$ urea, 2 $\mathrm{M}$ urea and no urea in the same buffer as above. Finally, the protein was dialyzed against the buffer $20 \mathrm{mM}$ Tris- $\mathrm{HCl} \mathrm{pH}$ $7.5,150 \mathrm{mM} \mathrm{NaCl}$ to remove DTT and concentrated to required concentration for further characterization in the same buffer.

\section{Analytical Ultracentrifugation}

Analytical ultracentrifugation (AUC) was carried out on an Optima XL-A analytical ultracentrifuge (Beckman Instruments, Palo Alto, CA) equipped with a 12-mm Epon double-sector cell in an An-60 Ti rotor. Sedimentation equilibrium runs were performed at $20^{\circ} \mathrm{C}$ at rotor speeds of 12,000 to $18,000 \mathrm{rpm}$ and protein concentrations of 0.1 to $0.5 \mathrm{mg} / \mathrm{ml}$. Average molecular masses were evaluated by using a floating base-line computer program SEGAL that adjusts the baseline absorbance to obtain the best linear fit of ln (absorbance) versus the square of the radial distance [35]. A partial specific volume $(v)$ of $0.73 \mathrm{~mL} / \mathrm{g}$ and a buffer density $(\rho)$ of $1.005 \mathrm{~g} / \mathrm{mL}$ was used.

\section{Electron Microscopy}

Protein samples were prepared for electron microscopy (EM) by negative staining with $2 \%$ uranyl acetate and at a protein concentration of $50 \mu \mathrm{g} / \mathrm{ml}$. They were analyzed and photographed on a Philips Morgagni 268D model transmission electron microscope with an acceleration voltage of 80 $\mathrm{kV}$.

\section{Circular Dichroism (CD) Measurements}

The CD measurements were acquired on an Aviv 62A DS spectropolarimeter. The far-ultraviolet spectra (200-250 $\mathrm{nm})$ were measured in a $1 \mathrm{~mm}$ path-length quartz cell at $4^{\circ} \mathrm{C}$. The spectra were normalized for concentration and path length to obtain the mean molar residue ellipticity $(\theta$, theta) after subtraction of the buffer contribution. The following formula was used to calculate the concentration independent theta $(\theta)$ values $\left(\operatorname{deg~} \mathrm{cm}^{2} \mathrm{dmol}^{-1}\right): \theta$ (observed) * MRW / [10 * path length $(\mathrm{cm}) *$ concentration $(\mathrm{mg} / \mathrm{ml})]$, where MRW mean residue weight (molecular mass of the peptide or protein divided by number of amino acids). Data analysis and the wavelength scan plots were performed with the pro Fit software package (QuantumSoft, Switzerland).

\section{Blue-Native PAGE (BN-PAGE) Electrophoresis}

For all the samples, $15 \mu \mathrm{g}$ of protein per gel lane was loaded and the protein samples were resuspended in the following buffer: $50 \mathrm{mM}$ BisTris- $\mathrm{HCl} \mathrm{pH} 7.0,500 \mathrm{mM} 6-$ aminocaproic acid and $50 \%$ glycerol. Samples were resolved on $5-12 \%$ gradient acrylamide gel overnight at $100 \mathrm{~V}$ using following anode $(50 \mathrm{mM}$ BisTris- $\mathrm{HCl} \mathrm{pH} \mathrm{7.00)}$ and cathode buffer $(50 \mathrm{mM}$ Tricine, $15 \mathrm{mM}$ Bistris- $\mathrm{HCl} \mathrm{pH} 7.0$ with $0.005 \%$ Coomassie G250) (Bio-Rad, USA) [36]. Estimates of molecular masses were obtained by comparison with high molecular weight standards for native gel electrophoresis (GE Healthcare Life Sciences, USA, range $669 \mathrm{kDa}$ to 66 $\mathrm{kDa}$ ). Proteins were visualized by staining with Coomassie Brilliant blue R250 (Bio-Rad, USA).

\section{RESULTS}

\section{Design Principles}

In this new design the SAPN have a tripartite structure composed of a trimeric coiled-coil head domain from laminin [30], the small, globular, trimeric $\beta$-propeller structure of the foldon domain [37] of fibritin followed by the pentameric coiled-coil domain from COMP [38] (Fig. 1C). The trimeric coiled-coil head domain can be cleaved off the peptide by using a thrombin cleavage site thus leaving a peptide chain that is composed of the foldon and the COMP domain only (Fig. 1B). This modular approach allowed us to test two different designs with two and three oligomerization domains, respectively, starting from the same gene construct.

According to this design, we engineered two versions of four different constructs 1 to 4 . The schematic representation and the amino acid sequences of these constructs is shown in Fig. 2. The C-terminal part of these constructs comprises the foldon domain (residues 55-82; blue), and the COMP domain (residues 85-126; green) separated by two glycine residues (brown) which act as a linker region, joining the foldon domain to the COMP domain. The glycine residues are used to provide flexibility between the two domains relative to each other. In the native coiled-coil domain of COMP the cysteine residues (Cys ${ }^{122}$ and $\mathrm{Cys}^{125}$ ) at the $\mathrm{C}$-terminal end form interchain disulfide bridges between the helices, as seen in the crystal structure of this protein [38]. The Nterminal part of the constructs includes a His-tag (residues 3 8; grey), the trimeric coiled-coil domain of laminin (residues 10-43; pink) and a thrombin cleavage site (residues 49-54; red). After thrombin cleavage, all the four constructs will have the residues glycine and serine from the thrombin cleavage site at their N-terminus.

In construct 2, a single aspartic acid to cysteine point mutation is introduced in the foldon domain at position 64; 
A
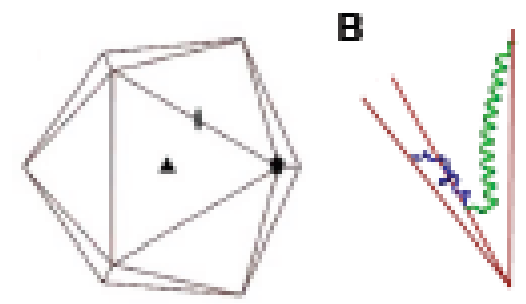
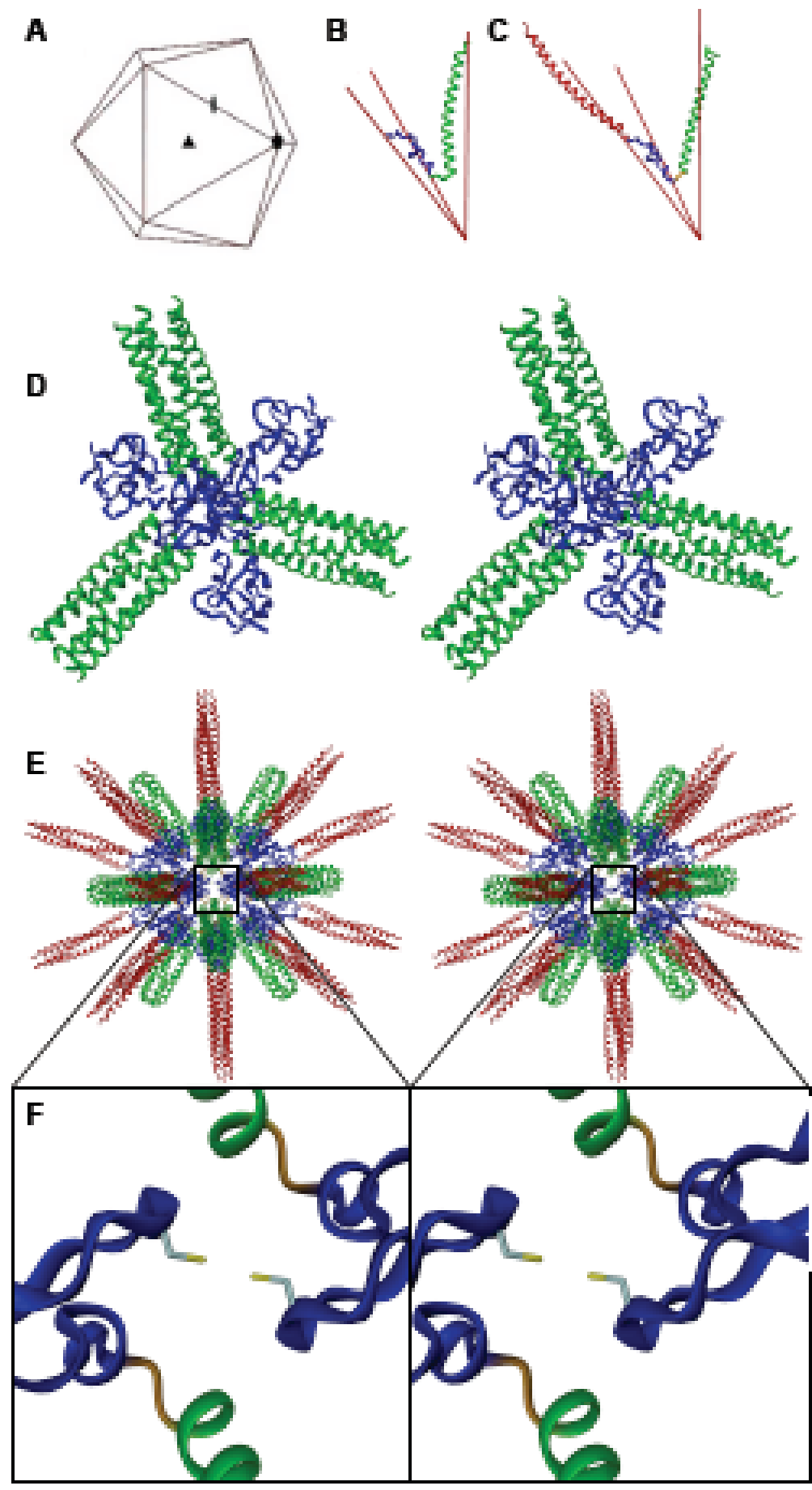

Fig. (1). (A) Icosahedron with the symbols of the three different symmetry elements. (B) Monomeric building block of the cleaved construct composed of the Foldon (blue) and the COMP (green) domains. (C) Monomeric building block of the uncleaved construct composed of the Laminin (red), Foldon (blue) and COMP (green) domains. (D) Stereo diagram of a model of an LCM-unit composed of the cleaved (FoldonCOMP) construct. (E) Stereo diagram of a model of an assembled nanoparticle with icosahedral symmetry composed of the uncleaved (Laminin-Foldon-COMP) construct. (F) Close-up stereo diagram of the modeled interaction of the two cysteine residues in a nanoparticle with icosahedral symmetry.

(bold and underscore in Fig. 2), otherwise the sequence is the same as that of construct 1 . The rationale behind this mutation is to introduce a disulfide bridge with the same residue of another peptide chain, to which it is symmetrically related by the two-fold rotation axis of the icosahedron. The intention is to cross-link two neighboring foldon trimers within the icosahedron that are related by the two-fold symmetry
(Fig. 1E and F) and hence to stabilize the SAPN. In the structure of the foldon domain [37], this cysteine residue is located in the loop region which connects the $\beta$ hairpin motif to the end of the last $\alpha$ helical segment, and hence it is not expected to stabilize the tertiary or quarternary structure of the foldon domain. Therefore, this mutation should not abolish the oligomeric state of the foldon domain. 

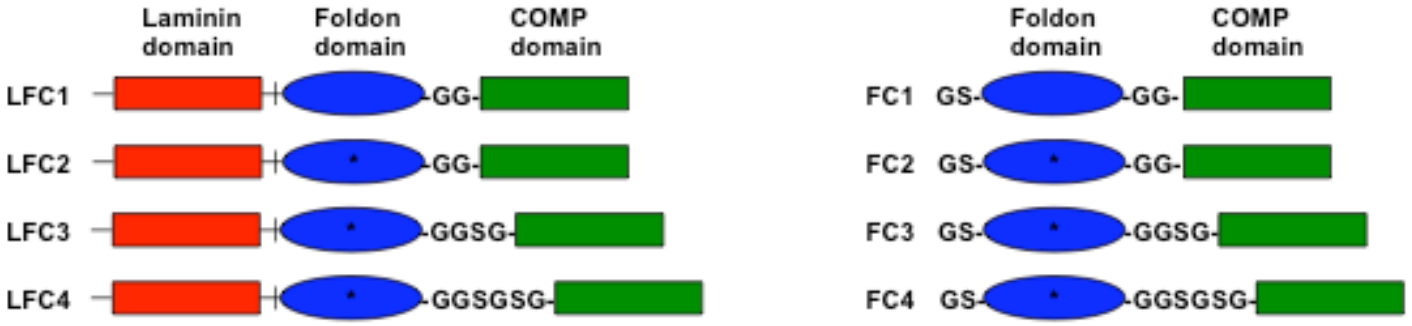

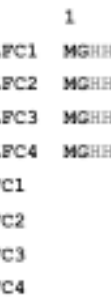

10

$20 \quad 30$

40

50

60

70

30

90

100

110

120

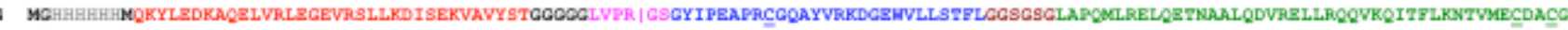

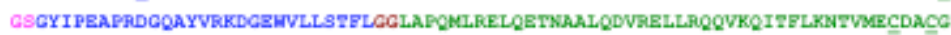
GSGYI PEAPRCGQAYVRKDGBWVLLSTFLGGLAPQNL.RELQETNARLQDVRELLRQQVKQTTFL.KNTVMECDACG GSGYTPEAPRCGQAYVRKDGEWVLLSTFLGGSGLAPQML.RELQETNAALQDVRELLRQQVKQTTFL.KWTVMECDACG GSGYIPEAPRCGQAYVRKDCSWVLLSFLLGSGSGLARCMLRELQETNALLDVRELLRQQVKQITFLKNTWMECDACO

Fig. (2). Top, schematic representation of constructs involved in this study. LFC1 to LFC4 represent the expressed constructs along with the $\mathrm{N}$-terminal vector coded laminin domain (red rectangle). Blue oval shape - foldon domain; green rectangle - COMP domain. The brown colored amino acids in between the foldon and COMP domain represent the linker residues. * represents the mutated amino acid aspartic acid (D) to cysteine (C) in the foldon domain. Vertical line (|) represents thrombin cleavage site. FC1 to FC4 represent the thrombin cleaved constructs LFC1 to LFC4. In FC1 to FC4 constructs, the two extra amino acids at the N-terminus which are result of thrombin cleavage, are highlighted pink. Bottom, amino acid sequence of LFC1 to LFC4 and FC1 to FC4 constructs. In LFC1 to LFC4 constructs, the N-terminal 54 amino acids are vector sequences coding for His-tag (grey), laminin domain (red) and thrombin recognition sequence (magenta). The green and blue colored amino acids represent the foldon and COMP domain, respectively. The mutated aspartic acid (D) to cysteine (C) amino acid in the foldon domain is indicated in bold and underlined, referred to as cysteine 1 . The C-terminal cysteine residues of the COMP domain are indicated in bold and underlined, referred to as cysteines 2 and 3.

In Raman et al. [26] we have argued that if the two different oligomeric domains of the monomeric building blocks fold properly, the monomeric building blocks will first assemble into 'even-units'. The number of monomers of such a unit will depend on the least common multiple (LCM) of the oligomeric state of the involved domains and hence we will call them "LCM-units". In our design, 15 monomers will form such an LCM-unit (Fig. 1D). One of the factors that is expected to determine the further assembly of these LCMunits into an icosahedral nanoparticle is the length of the linker region that connects the two oligomerization domains. To test this hypothesis, we extended the linker region between the foldon and the COMP domain from two to four (GGSG) and six residues (GGSGSG) yielding in the sequences of the third and fourth construct, respectively. The linker region between the laminin and the foldon domain on the other hand was kept constant in this design (5 glycine residues).

The four protein constructs were expressed in E. coli and then purified under denaturing conditions using a $\mathrm{Ni}^{2+}$ affinity column. All four purified, uncleaved samples were then also dialyzed against a thrombin cleavage buffer and cleaved by thrombin to give both, the uncleaved and cleaved versions of each of the four constructs LFC1 to LFC4 and FC1 to FC4. After thrombin cleavage, the protein was then purified, as described in the Materials and Methods section.

\section{Self-Assembly Properties of Cleaved Proteins FC1 to FC4}

First, the cleaved proteins FC1 to FC4 were studied for their self-assembly properties. Because of the presence of cysteine residues in these proteins they can form different combinations of intra- and interchain disulfide bridges during refolding. Therefore, we tried with two different refolding conditions. In the first refolding condition, the purified protein samples were refolded in a step-wise dialysis from 8 $\mathrm{M}$ to $0 \mathrm{M}$ urea under oxidizing conditions (regime 1). In the second refolding regime, the protein samples were refolded under reducing conditions ( $2 \mathrm{mM}$ DTT), followed by a final dialysis to oxidizing conditions (regime 2). The selfassembly behavior of the cleaved proteins under these two different refolding regimes was analyzed by Blue Native (BN)-PAGE and AUC to determine the molecular mass of the formed assemblies. The morphology of the oligomers was investigated by EM, and the secondary structure content was measured by CD-spectroscopy.

In the BN-PAGE for protein $\mathrm{FC} 1$, refolded by regime 1, we observed a band corresponding to pentamers $(41.9 \mathrm{kDa}$; Fig. 3 - lane 1). However, for the same sample refolded by regime 2 we observed an additional band which corresponds to decamers (83.8 kDa; Fig. 3 - lane 2). For protein FC2, refolded using regime 1 , we observed bands corresponding to trimers $(25.1 \mathrm{kDa})$, pentamers $(41.9 \mathrm{kDa})$ and LCM-units (125.6 kDa; Fig. 3 - lane 3). However, for regime 2 refolded samples we observed bands which correspond to pentamers and LCM-units, and we did not observe the trimeric band (Fig. 3 - lane 4). For protein FC3, refolded using regime 1, we observed bands corresponding to trimers $(25.5 \mathrm{kDa})$, pentamers (42.5 kDa) and LCM-units (127.5 kDa; Fig. 3 - lane $5)$. However, for regime 2 refolded samples, we observed bands which correspond to pentamers and LCM-units but again, no trimers were observed (Fig. 3 - lane 6). For FC4 


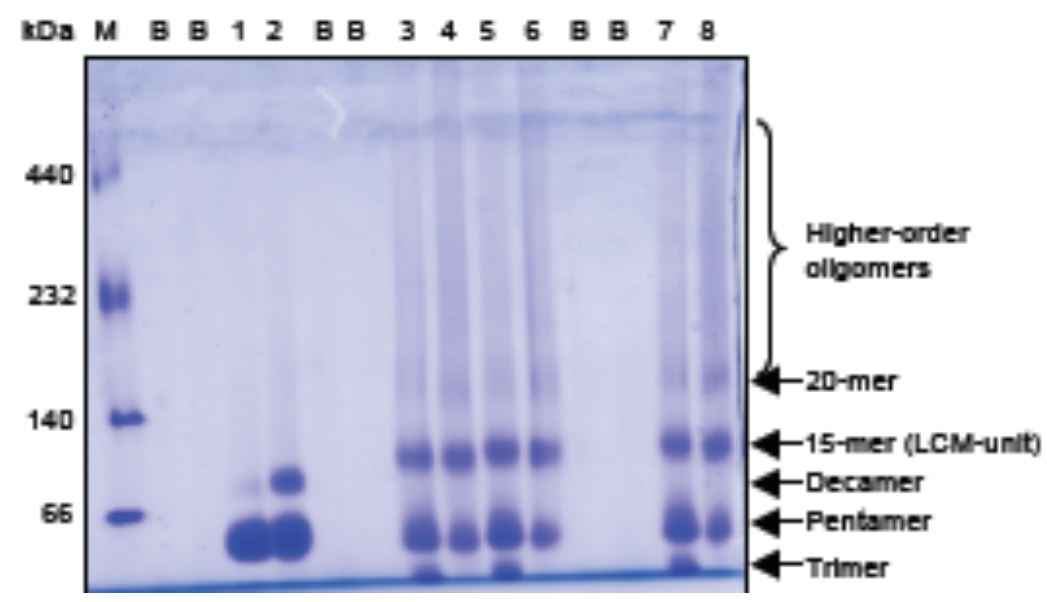

Fig. (3). 5 - 12\% gradient Blue Native (BN) PAGE of refolded cleaved (FC1 to FC4) proteins. The figure lanes are explained as follows: M High molecular weight native gel marker; analyzed in the buffer $20 \mathrm{mM}$ Tris $\mathrm{pH} 8.0,150 \mathrm{mM} \mathrm{NaCl}$ and $2 \mathrm{mM}$ DTT; lanes $1,3,5$ and 7 correspond to regime 1 refolded samples; lanes 2, 4, 6 and 8 correspond to regime 2 refolded samples; B- blank lanes.

protein, refolded using regime 1 , we observed bands corresponding to trimers $(25.9 \mathrm{kDa})$, pentamers $(43.2 \mathrm{kDa})$ and LCM-units (129.7 kDa; Fig. 3 - lane 7). However, for regime 2 refolded sample, we observed bands which correspond to pentamers (43.2 kDa) and LCM-units (129.7 kDa). We also observed an additional faint band which corresponds to 20mers (Fig. 3 - lane 8).

Molecular weight determination using analytical ultracentrifugation (AUC) for the FC1 samples under both conditions (regime 1 and 2) gave an average molecular mass which corresponds to pentamers (Table 1). The FC2 samples under both conditions (regime 1 and 2) gave molecular mass values which correspond to pentamers and 20-mers (Table 1). Based on the results of BN-PAGE, the observed 20-mers might well correspond to the mixture of LCM-units and possibly the LCM-units linked to pentamers through disulfide bridges (Fig. 7E). The regime 1 FC3 sample gave molecular mass values which correspond to pentamers and 20-mers (Table 1). On the other hand, regime 2 refolded samples gave molecular mass values which correspond to pentamers and 30-mers. The observation of 30-mers suggests nanoparticles composed of two LCM-units. The FC4 samples under both conditions (regime 1 and 2) gave molecular masses which correspond to pentamers and high molecular weight species. The high molecular weight species seem to be a mixed population of 20-mers and 30-mers (Table $\mathbf{1}$ ).

In the analysis of the morphology of the samples by EM, in both regime 1 (Fig. 4A) and regime 2 (Fig. 4B) refolded samples of FC1 protein, we observed short fragment-like structures. Based on the results of BN-PAGE and AUC, the observed short fragment-like structures might well correspond to pentamers and decamers. In both regime 1 (Fig. 4C) and regime 2 (Fig. 4D) of the FC2 samples, we observed nanoparticles not regular in shape. In addition, we observed a lot of background material. Based on the results of $\mathrm{BN}-$ PAGE and AUC, the observed nanoparticles which are not regular in shape might well correspond to LCM-units and 20-mers, and background material to trimers and pentamers. In both, regime 1 refolded samples of FC3 and FC4 (Fig. $4 \mathbf{E}$ and $\mathbf{F}$ ) and in regime 2 refolded samples of FC3 and FC4 (Fig. 4G and H), we observed nanoparticles but not with regular shape. Based on the BN-PAGE and AUC results of FC3 and FC4 proteins, the observed nanoparticles which are not regular in shape might well correspond to LCM-units and to the higher order oligomers.

We performed CD experiments in order to understand the secondary structural contents of the refolded samples because the laminin and COMP domain in the refolded samples are well characterized $\alpha$-helical coiled-coil proteins [3840]. Also, the foldon domain is a well studied $\beta$-hairpin motif structure [37]. The $\mathrm{CD}$ spectrum of both regime 1 and regime 2 refolded FC1 to FC4 proteins also showed a typical and similar $\alpha$-helical spectrum (Fig. 6). The cleaved proteins FC1 to FC4 showed a higher $\alpha$-helical spectrum compared to the $\alpha$-helical spectrum of LFC1 to LFC4 samples. The $\alpha$ helical content calculated from CD spectra using CD Pro software [41] (data not shown) showed 8 to $10 \%$ higher $\alpha$ helical content values compared to the theoretically calculated $\alpha$-helical content values (FC1, FC2 constructs - $58.7 \%$; FC3 construct - 57.1\%; FC4 construct - 55.7\%). The calculated $\beta$-sheet content from the CD spectra of FC1 to FC4 samples showed about $6 \%$ less than expected when compared to their theoretically calculated $\beta$-sheet content.

\section{Self-Assembly Properties of Uncleaved Proteins LFC1 to LFC4}

From the self-assembly studies of cleaved proteins FC1 to FC4, we understood that the cleaved proteins do not form

Table 1. Number of Monomers Per Particle for the Cleaved Proteins Measured by AUC

\begin{tabular}{|c|c|c|c|}
\hline & FC1 & FC2 & FC3 \\
\hline \hline Regime 1: Oxidizing & 5.3 & $5.3+19.8$ & $5.8+21.5$ \\
\hline Regime 2: Reducing & 5.3 & $6.0+21.4$ & $5.6+36.5$ \\
\hline
\end{tabular}


icosahedral SAPN composed of 60 monomers. We then studied the self-assembly properties of uncleaved proteins LFC1 to LFC4 (for construct details refer to Fig. 2).

For self-assembly studies, the purified samples of uncleaved proteins LFC1 to LFC4 in $8 \mathrm{M}$ urea were subjected to step wise dialysis of the urea under reducing condition and at low protein concentration (regime 2). The reason for reducing condition ( $2 \mathrm{mM}$ DTT) refolding is that the initial refolding experiment of LFC2 protein under oxidizing condition (regime 1) showed precipitating behavior of the protein at $4 \mathrm{M}$ urea concentration. Therefore, we decided to refold the proteins under reducing conditions only (regime 2 ).

\section{Initial Refolding Results of Uncleaved Protein}

Initially for $\mathrm{LFC} 1$ protein, the reducing condition refolding (regime 2) was done at $\mathrm{pH}$ 7.5. However, the refolded protein started to precipitate in refolding buffer containing 0 $\mathrm{M}$ urea and $2 \mathrm{mM}$ DTT (reducing condition). Subsequent change to the refolding buffer containing $0 \mathrm{M}$ urea and no DTT (oxidizing condition), the refolded protein precipitated heavily. From the EM analysis of sample in $0 \mathrm{M}$ urea with- out DTT, large aggregated structures with few individual nanoparticles are observed. The possible reason for precipitation in the refolding buffer containing $0 \mathrm{M}$ urea and $2 \mathrm{mM}$ DTT could be due to the $\mathrm{pI}$ of the protein which is calculated to be 6.29 . In addition, the observed heavy precipitation upon oxidizing condition could be due to linking of aggregates through interchain disulfide bridges. Therefore, the LFC1 samples were refolded at $\mathrm{pH} 8.0$ and 8.5 and analyzed in the refolding buffer containing $0 \mathrm{M}$ urea and $2 \mathrm{mM}$ DTT (reducing condition). The refolded samples are found to be stable in $0 \mathrm{M}$ urea containing $2 \mathrm{mM}$ DTT. From EM pictures, the refolded samples at $\mathrm{pH} 8.0$ and 8.5 are observed to form nanoparticles (data not shown).

To compare their self-assembly properties, all four proteins were refolded under reducing condition (regime 2) and the $\mathrm{pH}$ of the refolding buffer was kept at 8.0. The protein samples were refolded up to $0 \mathrm{M}$ urea containing $2 \mathrm{mM}$ DTT and analyzed in the same buffer using EM and AUC techniques. On the other hand the BN-PAGE results for LFC1, LFC2 and LFC4 proteins, showed no bands in the separating gel ( $5-12 \%$ gradient gel). This shows that the loaded pro-

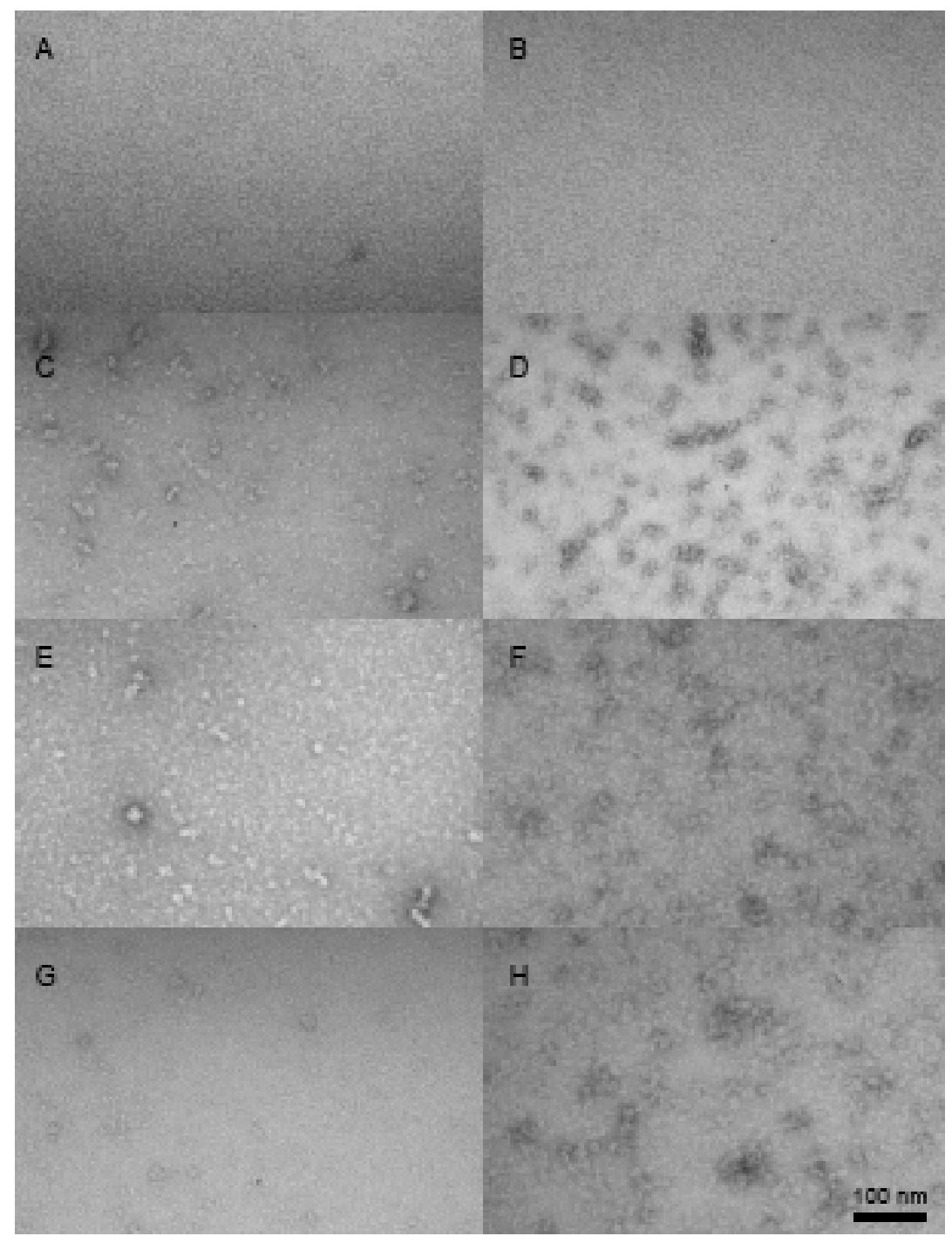

Fig. (4). Electron micrographs of constructs FC1, FC2, FC3, and FC4 (from top to bottom). The left column corresponds to refolding of the constructs according to regime 1 and the right column according to regime 2 , respectively. 


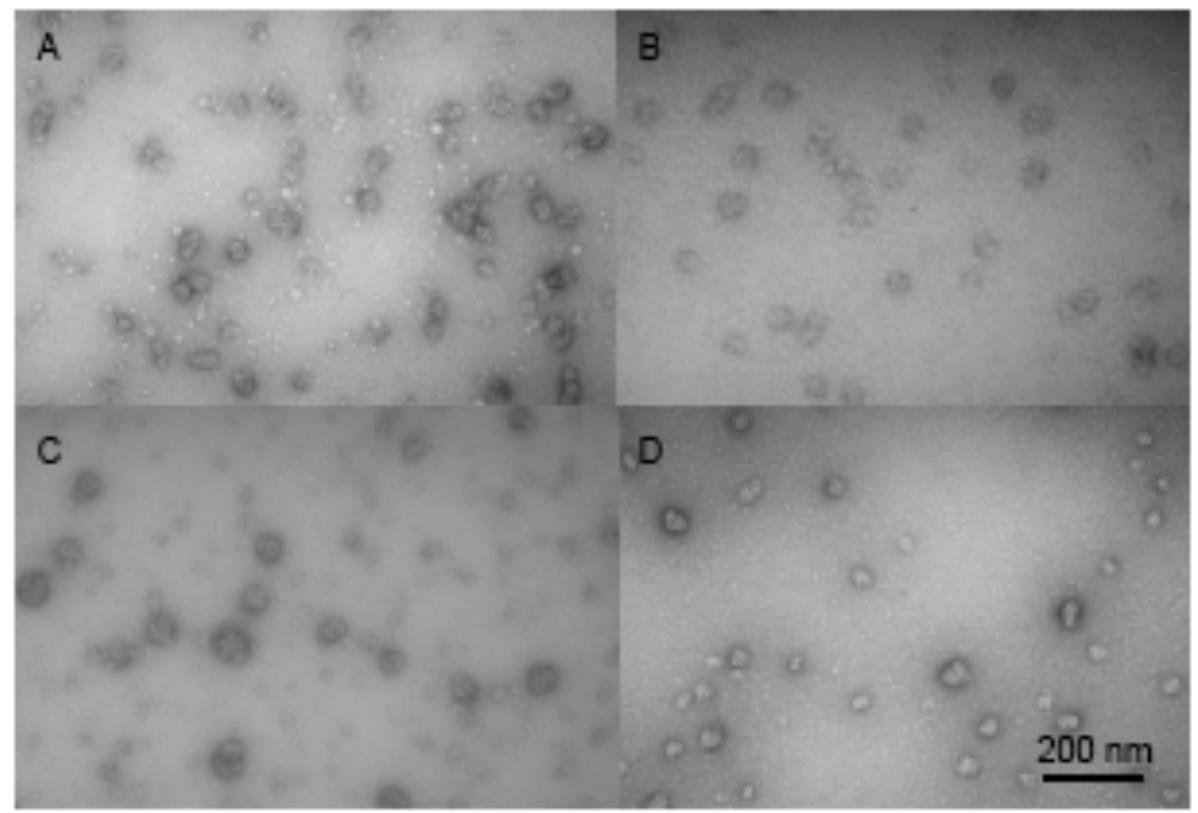

Fig. (5). Electron micrographs of refolded LFC1 to LFC4 proteins. A, LFC1; B, LFC2; C, LFC3; D, LFC4. All the four proteins were refolded under reducing condition after denaturation (regime 2).

teins got stuck in the stacking gel $(4.5 \%)$. The reason for this is most likely that resolution limit for the molecular weight of the gel doesn't go beyond about $500 \mathrm{kDa}$, and therefore larger species are not able to penetrate into the separating portion of the gel. For example, an LFC1 icosahedral nanoparticle with 60 peptide chains as shown in Fig. (1E) has a molecular weight of about $850 \mathrm{kDa}$.

The sedimentation equilibrium (SE) results from the AUC experiments for the LFC1 to LFC4 proteins refolded at $\mathrm{pH}$ 8.0, showed high molecular weight aggregates ranging from 1.7 MDa to 17.3 MDa.

For the LFC1 protein refolded at $\mathrm{pH} 8.5$, the SE results at lower speed $(2800 \mathrm{rpm})$ showed molecular weight values ranging from $611 \mathrm{kDa}$ to $7.2 \mathrm{MDa}$. The smallest molecular weight species $611 \mathrm{kDa}$ corresponds to a molecular weight of 3 LCM-units. At higher speed $(4800 \mathrm{rpm})$, the smallest molecular weight species showed mixed population of molecular weight values $632 \mathrm{kDa}$ (3 LCM-units) and $756 \mathrm{kDa}$ (3.6 LCM-units). Therefore, the observed low molecular weight species roughly correspond to icosahedral SAPN composed of 4 LCM-units. This is supported by the EM picture of LFC1 protein refolded at $\mathrm{pH} 8.5$, where we observed nanoparticles in the corresponding size range. The observed high molecular weight aggregates in all refolded proteins (LFC1 to LFC4) suggest some aggregation tendency of these nanoparticles.

From the EM pictures, all four proteins are observed to form nanoparticles in the size range that would be expected for icosahedral geometries: however, the nanoparticles are clearly not completely regular in their size and shapes (Fig. 5).

The uncleaved LFC1 to LFC4 samples showed a typical and similar $\alpha$-helical spectrum (Fig. 6). For uncleaved proteins LFC1 to LFC4, if both the laminin and COMP domain folds into an $\alpha$-helical structure, the theoretical $\alpha$-helical content will be the following: LFC1, LFC2 proteins - $61.4 \%$;
LFC3 protein - $60.5 \%$; LFC4 protein - $59.5 \%$. The calculated $\alpha$-helical content from CD spectra using CD Pro software [41] (data not shown) are similar to these theoretically calculated values. Also, the theoretically calculated $\beta$-sheet contents are similar to the calculated $\beta$-sheet content values from CD spectra. The above results support the formed $\alpha$ helical structures in both the laminin and COMP domain and the $\beta$-sheet structures in the foldon domain.

\section{DISCUSSION}

The cleaved proteins FC1 to FC4 were rationally designed to form SAPN with regular icosahedral symmetry, consisting of 60 monomeric building blocks. The basic idea for such a design was derived from the structure of small virus capsids, which usually display icosahedral symmetry. The peptide nanoparticles were designed to mimic such an icosahedral capsid geometry. VLPs are now widely used as a platform for rational vaccine design since the spacing of the epitopes on the surface of the VLPs makes them ideal repetitive antigen display systems. Therefore, also our peptide nanoparticles could possibly be used for vaccine design using the same concept of repetitive antigen display if they form particles with similar dimensions as VLPs. Computer modeling predicted that such particles would each have a diameter of about $17 \mathrm{~nm}$. The observed oligomeric forms of these proteins after refolding under regime 1 and regime 2 conditions are discussed in the following sections.

\section{Self-Assembly Result of Protein FC1}

The coiled-coil domain of COMP is known to form a very stable pentamer [42]. During refolding out of $8 \mathrm{M}$ urea, the coiled-coil domain of COMP will already form in urea concentrations that are moderately high. Formation of helices within the COMP domain will bring the two cysteines at the C-terminus of the COMP domain in close contact. For the sake of simplicity, the cysteine in the foldon domain will be called 1 and the two cysteines at the $\mathrm{C}$-terminus of COMP 

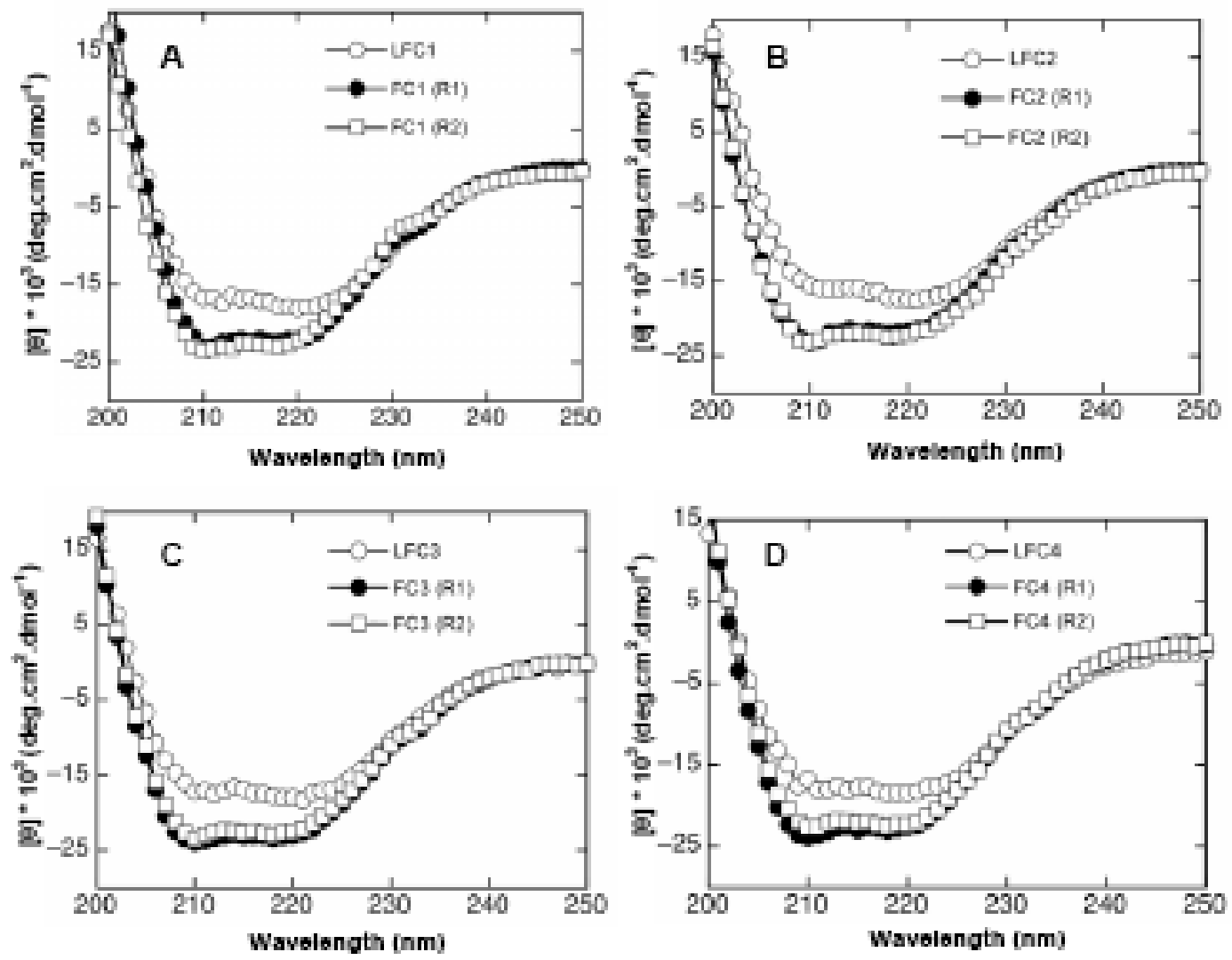

Fig. (6). Far-UV Circular Dichroism (CD) analysis of LFC1 to LFC4 and FC1 to FC4 samples. In A, B, C and D, open circles (o) represent the regime 2 refolded samples of LFC1, LFC2, LFC3 and LFC4, respectively; closed circles (•) represent the regime 1 (R1) and open squares (ם) represent the regime 2 (R2) refolded samples of FC1, FC2, FC3 and FC4, respectively. LFC1 to LFC4 samples were studied in the buffer $20 \mathrm{mM}$ Tris- $\mathrm{HCl} \mathrm{pH} 8.0,150 \mathrm{mM} \mathrm{NaCl}$ and $2 \mathrm{mM}$ DTT. FC1 to FC4 samples were studied in the buffer $20 \mathrm{mM}$ Tris- $\mathrm{HCl} \mathrm{pH} 7.5,150 \mathrm{mM}$ $\mathrm{NaCl}$. During measurement the protein concentration was kept at $0.1 \mathrm{mg} / \mathrm{ml}$.

domain will be called 2 and 3 (see Fig. 2), in order to explain the different combinations of intra- and interchain disulfide bridges that could form during refolding. Under oxidizing conditions with low protein concentration (regime 1) intrachain disulfide bridge formation will immediately and predominantly occur. Therefore, the pentamers observed in the regime 1 refolded sample of FC1 by native gel (Fig. 3; lane 3) and AUC (Table 1) suggest the folded COMP domain with the intrachain disulfide bridges (2-3). During regime 2 refolding, there is no possibility of forming either intra- or interchain disulfide bridges because of its refolding under reducing condition up to $0 \mathrm{M}$ urea. As a result, the cysteine residues will be in reduced state up to $0 \mathrm{M}$ urea: however, upon proceeding to oxidizing condition, they will form disulfide bridges. Therefore, the pentamers and decamers observed in regime 2 refolded samples (Fig. 3; lane 4) are presumably the intrachain disulfide linked pentamers (2-3) and two pentamers linked through interchain disulfide bridges (either 2-2, 2-3 or 3-3), respectively, depicted in Fig. (7B and $\mathbf{C}$ ).

The observed pentamers and decamers for $\mathrm{FC} 1$ protein suggest that the trimeric foldon domain is not folded in these structures because if the trimeric foldon domain folds along with the pentameric COMP domain they will assemble into 'LCM-units' (15-mers; Fig. 1D). In addition, it seems that the folding rate of the pentamer domain is faster than that of the trimer domain.

\section{Self-Assembly Result of Proteins FC2, FC3 and FC4}

For proteins FC2, FC3 and FC4, the LCM-units observed in both regime 1 and regime 2 refolding conditions (Fig. 3) are evidence of both properly folded COMP and properly folded foldon domain. In addition, the observed LCM-units support properly formed interchain disulfide bridges between the monomeric chains of the foldon domain (1-1) and between the monomeric chains of COMP domain (2-3). A schematic model of an LCM-unit is shown in Fig. (7D) (see also Fig. 1D).

FC2, FC3 and FC4 proteins have an additional cysteine residue in the foldon domain (1) in addition to the two cysteines in the COMP domain (2 and 3; see Fig. 2). During refolding under oxidizing conditions (regime 1) if the unfolded monomeric chains form either 1-2 or 1-3 intrachain disulfide bridges, this will lead to formation of pentamers or trimers. Therefore, the trimers observed in regime 1 refolded samples of FC2, FC3 and FC4 (Fig. 3; lane 3, 5 and 7) support the folded foldon domain with 1-2 or 1-3 intrachain disulfide bridges. During reducing condition refolding up to 0 $M$ urea (regime 2), the formation of 1-2 or 1-3 intrachain disulfide bridges is not possible, as this is supported by the absence of trimers in regime 2 refolded samples of FC2, FC3 and FC4 proteins (Fig. 3; lane 4, 6 and 8). A schematic model of a trimer is shown in Fig. (7A). The observation of trimers and LCM-units in the refolded samples of FC2, FC3 

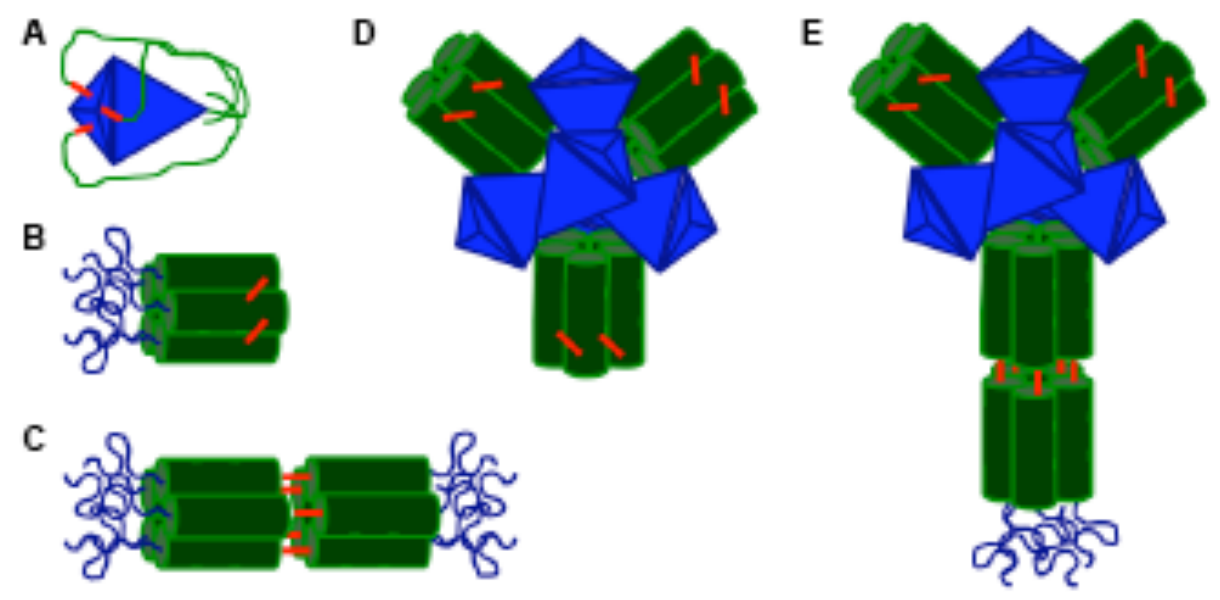

Fig. (7). Models of the different oligomerization states of the FC constructs. The foldon is shown in blue, the COMP is shown in green and disulfide bridges are depicted as red sticks. A) trimer (foldon folded, COMP unfolded) B) pentamer (COMP folded, foldon unfolded) $\mathbf{C}$ ) decamer (COMP folded, foldon unfolded) D) 15-mer (LCM-unit) E) 20-mer.

and FC4 proteins and not in the refolded samples of FC1 protein, suggests a more stable refolding of the previous three. Therefore, for proteins FC2, FC3 and FC4 the mutation of Asp (D) to Cys (C) (for sequence details see Fig. 2) in the foldon domain seems to stabilize the foldon domain in terms of its folding property.

Also, the BN-PAGE (Fig. 3) for proteins FC2, FC3 and FC4 refolded under both regime 1 (lanes 3, 5 and 7) and regime 2 conditions (lanes 4,6 and 8) shows an additional faint band corresponding to 20-mers. AUC results for proteins FC2, FC3 and FC4 suggest that the higher order oligomers (more than 15-mers) could be a mixture of 20-mers and 30-mers. The observation of 20-mers suggests the linked LCM-units and pentamers through interchain disulfide bridges. A schematic model of 20 -mers is shown in Fig. (7E). Furthermore, the observed pentamers in both the refolding conditions for all proteins FC1 to FC4 suggest that this is a common 'intermediate form' during the refolding process.

The LCM-units observed in proteins FC2, FC3 and FC4 support our design principles (Fig. 1, panel D). LCM-units are nanoparticles but not with icosahedral symmetry. Furthermore, the observed LCM-units failed to associate further into icosahedral nanoparticles.

\section{Uncleaved Proteins LFC1 to LFC4}

The regime 2 refolded proteins LFC1 to LFC4 were observed to form nanoparticles (see EM pictures of Fig. 5). The observation of nanoparticles in uncleaved proteins LFC1 to LFC4 can be explained on the basis of their domain architecture. The domain architecture of these uncleaved proteins comprises a 34 amino acid $\mathrm{N}$-terminal laminin domain followed by the foldon and the COMP domain (Fig. 2). The laminin domain was derived from the C-terminal region of the laminin $\beta$-chain of mouse (1750-1784 amino acids), and from the literature this region was known to be an $\alpha$-helical region with a heptad repeat pattern characteristic of coiledcoils [40, 43]. In our study, the formation of $\alpha$-helical structures in the laminin domain of the uncleaved proteins was supported by the $\alpha$-helical content calculated from the CD spectra of these proteins.
Kammerer, $\mathrm{R}$ et al. [30] showed that the laminin $\beta$-chain of residues 1700-1786, tends to associate into trimers. Therefore, the laminin domain in the uncleaved proteins, based on its $\alpha$-helical structure with the heptad repeat pattern represents a trimeric coiled-coil structure. In addition, the trimerization potential of the foldon domain $[44,45]$ will stabilize the formation of trimeric coiled-coil structure in the laminin domain and vice versa. Hence, the monomeric chain comprising the combined laminin-foldon trimeric oligomerization domain connected to the pentameric oligomerization domain of COMP mimics the design principles of our original SAPN design [26].

\section{CONCLUSION}

SAPN as described here can rather easily be functionalized in several ways: At either the $\mathrm{N}$ - or the $\mathrm{C}$-terminus the peptide chain can be extended with peptide sequences of a particular function like targeting peptides, cell penetrating peptides, nuclear localization sequences or epitopes from a pathogen to generate multifunctional nanoparticles for a wide variety of bio-medical applications. In particular, these SAPN can be used as repetitive antigen display system for synthetic vaccine design. The geometry of the SAPN and their resemblance to VLPs will trigger a strong immune response - a concept that is now increasingly exploited for producing novel vaccines that yield high titers of specific antibodies [46]. Our SAPN eliminate the need for virusbased designs, and allow for a higher flexibility in vaccine design compared to VLPs. Epitopes of any pathogen can readily be engineered onto the surface of the nanoparticle, thus allowing for the easy generation of a whole variety of different vaccines. Enveloped viruses such as HIV, SARS or influenza represent an ideal target as their surface proteins are characterized by trimeric coiled-coil proteins, which are the same building blocks that are used in our SAPN. Investigations of SAPN as potent immunogens and possible vaccine candidates are currently on their way in our laboratories and show very encouraging results.

\section{REFERENCES}

[1] Feynman R. There's plenty of room at the bottom. Eng Sci 1960; 22-36. 
[2] Quintana A, Raczka E, Piehler L, et al. Design and function of a dendrimer-based therapeutic nanodevice targeted to tumor cells through the folate receptor. Pharm Res 2002; 19(9): 1310-6.

[3] Wang SJ, Brechbiel M, Wiener EC. Characteristics of a new MRI contrast agent prepared from polypropyleneimine dendrimers, generation 2. Invest Radiol 2003; 38(10): 662-8.

[4] Bergey EJ, Levy L, Wang XP, et al. DC magnetic field induced magnetocytolysis of cancer cells targeted by LH-RH magnetic nanoparticles in vitro. Biomed Microdevices 2002; 4: 293-9.

[5] Jirak D, Kriz J, Herynek V, et al. MRI of transplanted pancreatic islets. Magn Reson Med 2004; 52(6): 1228-33.

[6] Hirsch LR, Stafford RJ, Bankson JA, et al. Nanoshell-mediated near-infrared thermal therapy of tumors under magnetic resonance guidance. Proc Natl Acad Sci USA 2003; 100(23): 13549-54.

[7] Reszka RC, Jacobs A, Voges J. Liposome-mediated suicide gene therapy in humans. Methods Enzymol 2005; 391: 200-8.

[8] ten Hagen TL. Liposomal cytokines in the treatment of infectious diseases and cancer. Methods Enzymol 2005; 391: 125-45.

[9] Zhu S, Oberdorster E, Haasch ML. Toxicity of an engineered nanoparticle (fullerene, C60) in two aquatic species, daphnia and fathead minnow. Mar Environ Res 2006; 62(Suppl): S5-9.

[10] Warheit DB, Laurence BR, Reed KL, Roach DH, Reynolds GA, Webb TR. Comparative pulmonary toxicity assessment of singlewall carbon nanotubes in rats. Toxicol Sci 2004; 77(1): 117-25.

[11] Whitesides GM, Grzybowski B. Self-assembly at all scales. Science 2002; 295(5564): 2418-21.

[12] Georgens C, Weyermann J, Zimmer A. Recombinant virus like particles as drug delivery system. Curr Pharm Biotechnol 2005; 6(1): 49-55.

[13] May T, Gleiter S, Lilie H. Assessment of cell type specific gene transfer of polyoma virus like particles presenting a tumor specific antibody Fv fragment. J Virol Methods 2002; 105(1): 147-57.

[14] Kramer RM, Li C, Carter DC, Stone MO, Naik RR. Engineered protein cages for nanomaterial synthesis. J Am Chem Soc 2004; 126(41): 13282-6.

[15] Ensign D, Young M, Douglas T. Photocatalytic synthesis of copper colloids from CuII by the ferrihydrite core of ferritin. Inorg Chem 2004; 43(11): 3441-6.

[16] Hosein HA, Strongin DR, Allen M, Douglas T. Iron and cobalt oxide and metallic nanoparticles prepared from ferritin. Langmuir 2004; 20(23): 10283-7.

[17] Flenniken ML, Willits DA, Brumfield S, Young MJ, Douglas T. The small heat shock protein cage from Methanococcus jannaschii Is a versatile nanoscale platform for genetic and chemical modification. Nano Lett 2003; 3(11): 1573-6.

[18] Flenniken ML, Liepold LO, Crowley BE, Willits DA, Young MJ, Douglas T. Selective attachment and release of a chemotherapeutic agent from the interior of a protein cage architecture. Chem Commun (Camb) 2005; (4): 447-9.

[19] Flenniken ML, Willits DA, Harmsen AL, et al. Melanoma and lymphocyte cell-specific targeting incorporated into a heat shock protein cage architecture. Chem Biol 2006; 13(2): 161-70.

[20] Seebeck FP, Woycechowsky KJ, Zhuang W, Rabe JP, Hilvert D. A simple tagging system for protein encapsulation. J Am Chem Soc 2006; 128(14): 4516-7.

[21] Paavola CD, Chan SL, Li Y, Mazzarella KM, McMillan RA, Trent JD. A versatile platform for nanotechnology based on circular permutation of a chaperonin protein. Nanotechnology 2006; 17: 11716.

[22] Kerfeld CA, Sawaya MR, Tanaka S, et al. Protein structures forming the shell of primitive bacterial organelles. Science 2005; 309(5736): 936-8.

[23] Lee LA, Wang Q. Adaptations of nanoscale viruses and other protein cages for medical applications. Nanomedicine 2006; 2(3): 13749.
[24] Drexler KE. Molecular engineering: an approach to the development of general capabilities for molecular manipulation. Proc Natl Acad Sci USA 1981; 78(9): 5275-8.

[25] Padilla JE, Colovos C, Yeates TO. Nanohedra: using symmetry to design self assembling protein cages, layers, crystals, and filaments. Proc Natl Acad Sci USA 2001; 98(5): 2217-21.

[26] Raman S, Machaidze G, Lustig A, Aebi U, Burkhard P. Structurebased design of peptides that self-assemble into regular polyhedral nanoparticles. Nanomedicine 2006; 2(2): 95-102.

[27] Burkhard P, Stetefeld J, Strelkov SV. Coiled coils: a highly versatile protein folding motif. Trends Cell Biol 2001; 11(2): 82-8.

[28] Delahay RM, Frankel G. Coiled-coil proteins associated with type III secretion systems: a versatile domain revisited. Mol Microbiol 2002; 45(4): 905-16.

[29] McAlinden A, Smith TA, Sandell LJ, Ficheux D, Parry DA, Hulmes DJ. Alpha-helical coiled-coil oligomerization domains are almost ubiquitous in the collagen superfamily. J Biol Chem 2003; 278(43): 42200-7.

[30] Kammerer RA, Antonsson P, Schulthess T, Fauser C, Engel J. Selective chain recognition in the C-terminal alpha-helical coiledcoil region of laminin. J Mol Biol 1995; 250(1): 64-73.

[31] Brandenberger R, Kammerer RA, Engel J, Chiquet M. Native chick laminin-4 containing the beta 2 chain (s-laminin) promotes motor axon growth. J Cell Biol 1996; 135(6 Pt 1): 1583-92.

[32] Laemmli UK. Cleavage of structural proteins during the assembly of the head of bacteriophage T4. Nature 1970; 227(5259): 680-5.

[33] Towbin H, Staehelin T, Gordon J. Electrophoretic transfer of proteins from polyacrylamide gels to nitrocellulose sheets: procedure and some applications. Proc Natl Acad Sci USA 1979; 76(9): 43504.

[34] Burnette WN. "Western blotting": electrophoretic transfer of proteins from sodium dodecyl sulfate--polyacrylamide gels to unmodified nitrocellulose and radiographic detection with antibody and radioiodinated protein A. Anal Biochem 1981; 112(2): 195-203.

[35] Machaidze G, Lustig A. SEGAL, a semi-automatic program for fitting sedimentation equilibrium patterns from analytical ultracentrifugation. J Biol Phys Chem 2006; 6: 91-102.

[36] Schagger H. Blue-native gels to isolate protein complexes from mitochondria. Methods Cell Biol 2001; 65: 231-44.

[37] Tao Y, Strelkov SV, Mesyanzhinov VV, Rossmann MG. Structure of bacteriophage T4 fibritin: a segmented coiled coil and the role of the C-terminal domain. Structure 1997; 5(6): 789-98.

[38] Malashkevich VN, Kammerer RA, Efimov VP, Schulthess T, Engel J. The crystal structure of a five-stranded coiled coil in COMP: a prototype ion channel? Science 1996; 274(5288): 761-5.

[39] Barlow DP, Green NM, Kurkinen M, Hogan BL. Sequencing of laminin B chain cDNAs reveals C-terminal regions of coiled-coil alpha-helix. Embo J 1984; 3(10): 2355-62.

[40] Sasaki M, Kato S, Kohno K, Martin GR, Yamada Y. Sequence of the cDNA encoding the laminin B1 chain reveals a multidomain protein containing cysteine-rich repeats. Proc Natl Acad Sci USA 1987; 84(4): 935-9.

[41] Sreerama N, Woody RW. Estimation of protein secondary structure from circular dichroism spectra: comparison of CONTIN, SELCON, and CDSSTR methods with an expanded reference set. Anal Biochem 2000; 287(2): 252-60.

[42] Guo Y, Kammerer RA, Engel J. The unusually stable coiled-coil domain of COMP exhibits cold and heat denaturation in 4-6 M guanidinium chloride. Biophys Chem 2000; 85(2-3): 179-86.

[43] Paulsson M, Deutzmann R, Timpl R, Dalzoppo D, Odermatt E, Engel J. Evidence for coiled-coil alpha-helical regions in the long arm of laminin. Embo J 1985; 4(2): 309-16.

[44] Stetefeld J, Frank S, Jenny M, et al. Collagen stabilization at atomic level: crystal structure of designed (GlyProPro)10foldon. Structure 2003; 11(3): 339-46. 
[45] Papanikolopoulou K, Teixeira S, Belrhali H, Forsyth VT, Mitraki A, van Raaij MJ. Adenovirus fibre shaft sequences fold into the native triple beta-spiral fold when N-terminally fused to the bacteriophage T4 fibritin foldon trimerisation motif. J Mol Biol 2004; 342(1): 219-27.
[46] Lechner F, Jegerlehner A, Tissot AC, et al. Virus-like particles as a modular system for novel vaccines. Intervirology 2002; 45(4-6): 212-7.

(C) Raman et al.; Licensee Bentham Open.

This is an open access article licensed under the terms of the Creative Commons Attribution Non-Commercial License (http://creativecommons.org/licenses/by-nc/3.0/) which permits unrestricted, non-commercial use, distribution and reproduction in any medium, provided the work is properly cited. 